\title{
INTERVENCIÓN CONDUCTUAL EN UN CASO DE BAJO ESTADO DE ÁNIMO
}

\section{BEHAVIORAL INTERVENTION IN CASE OF LOW MOOD}

\author{
Marta Ortega Otero, Mateusz Kendzierski, Ma Elena Aranda Rubio \\ Universidad Autónoma de Madrid (España)
}

Cómo referenciar este artículo/How to reference this article:

Ortega Otero, M., Kendzierski M. y Aranda Rubio, M. E. (2015). Intervención Conductual en un Caso de Bajo Estado de Ánimo. Revista de Psicoterapia, 26(102), 175-188.

\begin{abstract}
Resumen
El presente trabajo describe el tratamiento llevado a cabo con un varón de 27 años que presenta sintomatología ansioso-depresiva. A partir del análisis funcional se decidió combinar distintas técnicas cognitivo-conductuales, tales como la activación conductual, el entrenamiento en toma de decisiones, el entrenamiento en habilidades sociales, la reestructuración cognitiva, técnicas de manejo de la ira y pautas de higiene del sueño. Tras cinco meses de intervención, seconcluye su eficacia en el control de la sintomatología ansioso-depresiva. Concretamente, se apreció un cambio significativo en la puntuación del BDI-II, en el número y el tipo de actividades desarrolladas, en la reducción de las rumiaciones, la mejora de la calidad del sueño y las verbalizaciones del cliente. Los beneficios terapéuticos se mantuvieron en la sesión de seguimiento.

Palabras Clave: sintomatología ansioso-depresiva, terapia cognitivo-conductual, análisis funcional, experimento de caso único, estudios de casos.
\end{abstract}

\begin{abstract}
This paper describes the treatment carried out with a 27 year old male presenting anxious-depressive symptomatology. Based on functional analysis it was decided to combine different cognitive-behavioral techniques like behavioral activation, training in decisionmaking, social skills training, cognitive restructuring, anger management and sleep hygiene guidelines. After five months of intervention, their effectiveness is concluded in the control of anxiety-depressive symptomatology. Specifically, a significant change was observed in the score of the BDI-II, in the number and type of activities performed, reduction of ruminations, improvement of quality of sleep and the patient verbalization. The therapeutic benefits were maintained in the follow-up session.

Keywords: anxiety-depression symptomatology, behavioural-cognitive therapy, functional analysis, single-case experiment, case studies.
\end{abstract}




\section{Introducción}

La prevalencia de los trastornos mentales en la Atención Primaria es de entre el 20 y el 55\% (Aragonés, 2004; Serrano-Blanco, 2010; Grandes, 2011). Se ha estimado que una cuarta parte de los pacientes que acuden a la consulta de Atención Primaria han tenido un trastorno mental en el año anterior y, en particular, problemas de ansiedad y/o depresión (Serrano-Blanco, 2010; Grandes, 2011; Díaz, 2001; Borrell-Carrió, 2003).

La coexistencia de la ansiedad y la depresión es más habitual de lo que podría parecer (Vallejo, 2000). En concreto, los trastornos de adaptación son de los más comunes, ya que muchos pacientes presentan signos y síntomas de ansiedad y depresión al tratar de adaptarse a un contexto cambiante o nuevo (Grandes, 2011; Hornillos, 2009).

Los manuales diagnóstico más utilizados en la práctica clínica, como el DSMIV-TR (American Psychiatric Association, 2000), al igual que la CIE-10 (Organización Mundial de la Salud, 1992), contemplan la posibilidad del diagnóstico de un trastorno conjunto que incluya síntomas de ansiedad y depresión. Ninguno de los dos sistemas llega a precisar su diagnóstico más allá de la sugerencia (como hace la CIE-10) de que deben estar presentes síntomas de ambos trastornos, sin que el conjunto de síntomas por separado sea suficiente para poder diagnosticar uno de ellos.

En la reciente publicación del manual DSM-V (American Psychiatric Association, 2013), se ha optado por eliminar la categoría diagnóstica de trastorno mixto ansioso-depresivo y a cambio se incluye el especificador "con síntomas de ansiedad", tanto para los trastornos depresivos como para los bipolares.

A pesar de la dificultad diagnóstica, son varios los estudios que han tratado de valorar la eficacia de la intervención cognitivo-conductual en este tipo de casos (Echeburúa, 2000; Jauregui, 2015), llegando a conseguir también evidencias a largo plazo (Echeburúa, 2006).

La eficacia de la terapia psicológica, y en concreto de la terapia cognitivoconductual, se ha mostrado igual o superior que los tratamientos farmacológicos y con una mayor efectividad a largo plazo en el tratamiento de los trastornos afectivos y de ansiedad más comunes (Hollon, 2006, Butler, 2006, Spielmans, 2011). En particular, los beneficios de los enfoques psicológicos incluyen una reducción en los síntomas asociados con la ansiedad y la depresión, disminución en el riesgo de recaídas, estabilidad del efecto del tratamiento a largo plazo, altas tasas de recuperación, prevención de la cronicidad y disminución de los costes -en términos de consultas médicas, el uso de fármacos o bajas laborales- (Richards, 2009; Van Orden 2009; Hunsley, 2007).

La intervención que se ha propuesto para este cuadro clínico, y que ha tenido mayor apoyo empírico, es la combinación de técnicas cognitivo-conductuales utilizadas en los trastornos de ansiedad y en los de la depresión. En concreto, las técnicas para el control de la ansiedad (relajación muscular y respiración controla- 
da), la reestructuración cognitiva, la activación conductual o implicación en actividades gratificantes y el entrenamiento en habilidades sociales y de solución de problemas (Pérez, 2003, Echeburúa, 2000, 2006).

El presente trabajo tiene como objetivo describir la intervención cognitivoconductual en el caso de un sujeto que presenta síntomas ansioso-depresivos, atendido en el Centro de Psicología Aplicada de la Universidad Autónoma de Madrid.

\section{Presentación del caso}

\section{Identificación del paciente y motivo de consulta}

M. (nombre ficticio) es un hombre de 27 años, estudiante de la Universidad Autónoma de Madrid. Sus motivos de consulta y, por tanto objetivos de evaluación, son el bajo estado de ánimo y la ansiedad. Concretamente acude por temblores, dificultad para respirar, rumiaciones, visión de túnel y dificultades de mantenimiento del sueño.

Se relaciona el origen de su problema de ansiedad con la incertidumbre que sintió hace seis años ("no sabía qué iba a hacer con mi vida") así como con el fracaso que supuso un suspenso que le impidió pasar de curso y con la presión que sintió por su pareja ("me cuestionaba, ella era extremadamente trabajadora").

Además, M. dejó de tocar la guitarra porque se lesionó los brazos, dejó su grupo de música y perdió parte del contacto con sus amigos y amigas, por dedicar tiempo a la relación de pareja.

M. mantiene su crisis vital y siente incertidumbre respecto a su actual relación de pareja, lo que le provoca una gran insatisfacción al no encontrar solución.

Para tranquilizarse y distraerse, M. se mete en la cama, lee y hace deporte. Cuenta que, además, su apetito fluctúa porque cuando está más nervioso, le cuesta poder controlarse durante la ingesta.

En la segunda sesión, M. comunica su ruptura de pareja y expresa verbalizaciones de desesperanza y tristeza como "no tiene sentido nada", "no voy a superar el dolor", "estoy solo", que aparecen de forma recurrente.

Ha reducido el contacto con sus amigos por temor a caer mal y además dice tener dificultades para expresar su enfado o comunicar sus necesidades.

\section{Estrategias de evaluación}

La evaluación inicial del problema consistió en una entrevista abierta para recabar la información necesaria para, posteriormente, establecer el análisis funcional. Se requirieron cinco sesiones en las que también se utilizaron auto-registros para determinar la interferencia a nivel laboral, familiar y social

Su estado de ánimo se evaluó, así mismo, mediante la administración del Inventario de Depresión de Beck-II (BDI-II; Beck et al., 1996). En este caso se empleó la versión en español baremada con muestras clínicas y no clínicas (Sanz 
et al., 2003; Sanz et al., 2003) y M. obtuvo una puntuación de 28 (depresión moderada).

Se administró también un modelo de Historia Biográfica utilizado en el Centro de Psicología Aplicada de la Universidad Autónoma de Madrid, que incluye preguntas sobre el motivo de consulta, expectativas respecto a la terapia, relaciones sociales de la persona, familia, aspectos laborales, etc.

Durante las sesiones de evaluación, y con periodicidad semanal, se pidió un auto-registro de bienestar y otro del cumplimiento de las actividades gratificantes, siendo éste el problema más característico del cliente. El auto-registro del bienestar se mantuvo durante el tratamiento para medir la evolución del cliente y la eficacia del tratamiento aplicado. Además, en distintos momentos del proceso (evaluación y tratamiento) se evaluaron mediante auto-registros las siguientes variables: pensamientos irracionales, rumiaciones, respuestas asertivas en la interacción, el sueño, así como, la respiración diafragmática. Tales variables permitieron tanto confirmar las hipótesis funcionales de la fase de evaluación, como controlar la aplicación del tratamiento en el trabajo inter-sesiones.

\section{Formulación clínica del caso}

\section{Variables disposicionales}

Entre las variables disposicionales podemos destacar que el cliente es un hombre de 27 años que, hace dos años, dejó de tocar la guitarra porque se lesionó los brazos, dejó su grupo de música y perdió el contacto con sus amigos. Presenta ideas irracionales del tipo: "¿a quién le importan mis problemas?”, "les voy a caer mal", "no tiene sentido nada", "no voy a superar el dolor", "estoy solo", "me voy a quedar solo" y alteración del sueño.

\section{Análisis Funcional}

Con los datos recogidos en la evaluación, se hipotetiza que el origen de las conductas problema de M. puede estar relacionado con el malestar generado por el fracaso que supuso suspender el examen de la asignatura que le impidió pasar de curso, la pérdida de reforzadores (ruptura de pareja, amigos, grupo de música, etc.) y con la incertidumbre propia de su situación vital. Por condicionamiento clásico, los pensamientos sobre su valía, sobre el pasado, su expareja y todo lo relacionado con ella también acaban por generarle malestar.

En cuanto a las hipótesis de mantenimiento se concluye que en las relaciones sociales, por influencia de las creencias irracionales y el déficit en habilidades sociales, su conducta es pasiva. Tal patrón se mantiene por el alivio del malestar que le supone a corto plazo. Sus ideas irracionales de lectura de pensamiento, junto con el malestar que le produce estar en el barrio donde vive su expareja, dan lugar a que M. ingiera comida rápida para aliviar su malestar y conseguir la satisfacción que, en sí mismo, produce comer. Ante los pensamientos sobre su pasado y sobre el 
futuro, que le generan malestar, aparecen las rumiaciones y los contraargumentos ("qué hubiera pasado si...", “qué hago con mi vida?"," no sé qué hacer") que se mantienen por el alivio del malestar a corto plazo. Estas rumiaciones y contraargumentaciones, a su vez, se relacionan con conductas como meterse en la cama, que reducen su malestar a corto plazo. M. tampoco tiene un trabajo estable ni satisfactorio y evita la búsqueda de un nuevo empleo por el alivio del malestar a corto plazo que le supone postergar al anticipar el fracaso si no lo encontrase.

En la Figura 1 puede verse un esquema resumen de las hipótesis de origen y mantenimiento.

Hipótesis de origen:

$$
\begin{gathered}
\text { El: fracaso } \rightarrow \mathrm{RI}: \text { malestar } \\
\text { EC: suspender el examen } \rightarrow \mathrm{RC}: \text { malestar } \\
\text { El: pérdida } \rightarrow \mathrm{RI}: \text { malestar } \\
\text { EC: ruptura de pareja } \rightarrow \text { RC: malestar } \\
\text { ED: novia, amigos, grupo de músiea, ete. } \rightarrow \text { Rop: aetividades gratifieantes } \rightarrow \\
\text { G: } \\
\text { El: incertidumbre } \rightarrow \text { RI: malestar } \\
\text { EC: pensamientos sobre el pasado y el futuro } \rightarrow \text { RC: malestar }
\end{gathered}
$$

Hipótesis de mantenimiento:

ED: situación interpersonal $\rightarrow$ Rop: conducta pasiva (no expresa su malestar ni sus necesidades) $\rightarrow$ C: alivio del malestar (R-)

ED: antes de acostarse $\rightarrow$ Rop: rumiaciones y contraargumentaciones $\rightarrow$ C: alivio del malestar (R-)

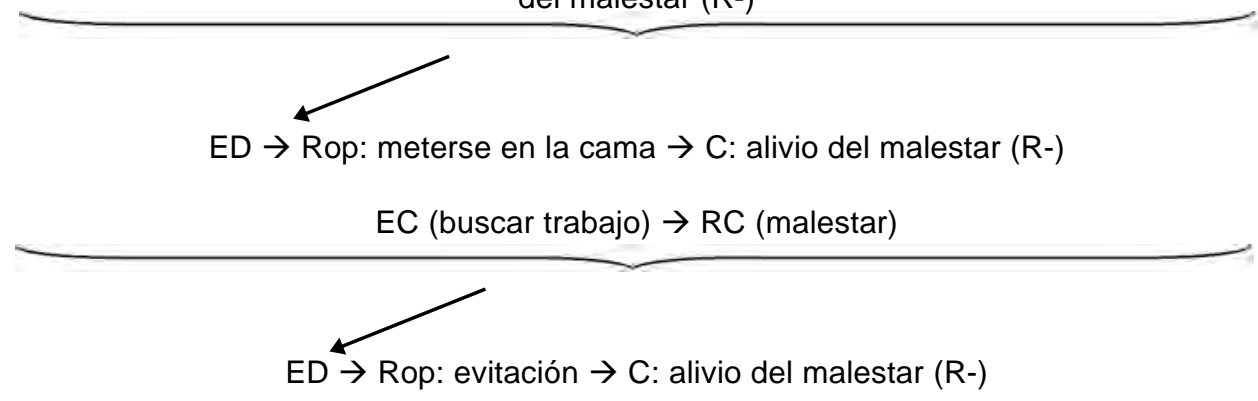

Figura 1. Origen y mantenimiento de las conductas problema. EI: estímulo incondicionado; RI: respuesta incondicionada; EC: estímulo condicionado; RC: repuesta condicionada; Ed: estímulo discriminativo; Rop: respuesta operante; R-: refuerzo negativo; $\mathrm{R}+$ : refuerzo positivo.

\section{Tratamiento}

\section{Elección del tratamiento}

Dada la formulación clínica del caso, se anticipó que en el tratamiento se utilizarían las siguientes técnicas cognitivo-conductuales (Echeburúa, 2000; Pérez, 
2003): (1) activación conductual (planificación y monitorización de actividades gratificantes), (2) entrenamiento en toma de decisiones (descripción del trabajo y planificación de la búsqueda de trabajo), (3) reestructuración cognitiva de las ideas irracionales, (4) pautas básicas de higiene del sueño, (5) entrenamiento en habilidades asertivas (expresión del malestar, hacer y rechazar peticiones), (6) técnicas de manejo de la ira (tiempo fuera, respiración diafragmática, reestructuración cognitiva) y (7)control estimular.

\section{Aplicación del tratamiento}

El tratamiento tuvo una duración de diecinueve sesiones que se llevaron a cabo en el transcurso de cinco meses. Las sesiones fueron semanales y de una hora de duración. Las cinco primeras sesiones fueron de evaluación, la sexta sesión se dedicó a la explicación del análisis funcional, doce sesiones fueron de tratamiento y el seguimiento se realizó en una única sesión, a los quince días.

Tras las primeras cinco sesiones de evaluación se explicó el análisis funcional para justificar y proponer los objetivos que se iban a perseguir y las técnicas que se llevarían a cabo (véase Tabla 1).

Se acordaron con el cliente los siguientes objetivos terapéuticos: a nivel general, aumentar el estado de ánimo (a niveles previos a hace dos años) y disminuir la ansiedad ante determinadas estímulos. En cuanto a los objetivos específicos se postularon los siguientes: (a) aumentar la tasa de actividades gratificantes, (b) modificar las ideas irracionales sobre su valía, sobre las relaciones interpersonales y el sentido de la vida, (c) mejorar el patrón de sueño, (d) reducir las rumiaciones y contraargumentaciones, (e) mejorar las habilidades interpersonales y (f) aprender nuevas estrategias de afrontamiento (ante la ira y la ansiedad).

Tras la explicación del análisis funcional, se aplicó la técnica de activación conductual programando actividades gratificantes, comprobando su cumplimiento e insistiendo en la relación que existe entre las actividades que se realizan y el estado de ánimo. Además el consultante completaba auto-registros de bienestar para aumentar la probabilidad de que M. centrase su atención en los acontecimientos positivos que le ocurrían a lo largo de la semana.

En cuanto a la búsqueda de trabajo, en la sexta sesión se pidió al cliente que identificara las características de su trabajo ideal y se fomentó la toma de decisiones entre trabajar por cuenta ajena y trabajar por cuenta propia. Después de valorar las ventajas y desventajas de cada opción, M. se decantó por el emprendimiento y, en concreto, la creación de su propia empresa. En ese momento, se le invitó a que pensara en los pasos que le permitieran acercarse a su objetivo. En la sexta sesión se utilizó la técnica de control estimular para reducir la impulsividad de sus conductas con la comida.

Se utilizó también, en la undécima sesión, la reestructuración cognitiva, y el consultante realizó auto-registros de sustitución de ideas irracionales por otras más realistas durante las ocho sesiones siguientes. 
A partir de la decimocuarta sesión, y con el objetivo de aprender habilidades asertivas, se entrenaron las habilidades de expresión de emociones negativas, hacer críticas y hacer peticiones. Concretamente, se realizaron distintos role-playing (incluyendo el modelado por parte de la terapeuta), se entregaron lecturas y el consultante tuvo que realizar auto-registros de interacciones para aplicar y comprobar lo aprendido en las sesiones.

También se llevó a cabo el entrenamiento en respiración diafragmática. para que M. pudiera aplicar una estrategia adaptativa en los momentos de mayor activación.

En cuanto a sus dificultades de sueño, en la séptima sesión se pidió que realizara un diario del sueño para poder evaluarlo con detalle. En la novena sesión se dieron pautas de higiene de sueño y, en las sucesivas, se comprobó que el sueño iba mejorando a medida que se aplicaban las pautas enseñadas.

Ante algunas ideas irracionales sobre sexualidad, que aparecieron de forma esporádica en la decimoquinta y decimonovena sesión, la terapeuta reestructuró estas ideas y utilizó la psicoeducación.

M. verbaliza en la sesión de seguimiento que ha utilizado las habilidades sociales aprendidas y que sigue utilizando la respiración como estrategia. En esta sesión se hace prevención de recaídas recordando las habilidades y analizando situaciones difíciles que podrían presentarse en el futuro.

Tabla 1.

Plan de tratamiento

\begin{tabular}{llll}
\hline Sesión & Objetivos & Técnicas & Tareas entre sesiones \\
\hline $1-5$ & Evaluar & $\begin{array}{l}\text { Entrevista abierta } \\
\text { Reestructuración } \\
\text { cognitiva } \\
\text { Cuestionario }\end{array}$ & $\begin{array}{l}\text { Historia biográfica } \\
\text { Auto-registros } \\
\text { BDI-II }\end{array}$ \\
\hline 6 & Explicar el análisis funcional, & Entrevista abierta & Auto-registro de bienestar \\
& acordar los objetivos y & Reestructuración & Escribir el análisis funcional \\
& anticipar la intervención & cognitiva & Actividades gratificantes \\
& que se llevará a cabo & Activación conductual & Identificación de las \\
& & Control estimular & características del trabajo ideal \\
& & & Toma de decisiones respecto al \\
& & trabajo \\
\hline 7 & Reducir rumiaciones, reducir la & Reestructuración & AR de bienestar \\
desesperanza y la incertidumbre sobre & cognitiva & Escribir el AF \\
su futuro & Activación conductual & Actividades gratificantes \\
Aumentar actividades gratificantes y & Entrenamiento en & Esquema sobre su trabajo \\
& aprender estrategias de afrontamiento & asertividad & Diario de sueño \\
más adaptativas & & \\
Planificar la búsqueda de trabajo. & & \\
Aprender habilidades asertivas & &
\end{tabular}




\begin{tabular}{|c|c|c|c|}
\hline 8 & $\begin{array}{l}\text { Reducir rumiaciones, reducir la } \\
\text { desesperanza y la incertidumbre sobre } \\
\text { su futuro } \\
\text { Aumentar actividades gratificantes y } \\
\text { aprender estrategias de afrontamiento } \\
\text { más adaptativas } \\
\text { Planificar la búsqueda de trabajo. } \\
\text { Aprender habilidades asertivas }\end{array}$ & $\begin{array}{l}\text { Activación conductual } \\
\text { Entrenamiento en } \\
\text { asertividad }\end{array}$ & $\begin{array}{l}\text { AR de bienestar } \\
\text { Escribir el AF } \\
\text { Actividades gratificantes }\end{array}$ \\
\hline 9 & $\begin{array}{l}\text { Reducir rumiaciones, reducir } \\
\text { la desesperanza y la incertidumbre } \\
\text { sobre su futuro } \\
\text { Aumentar actividades gratificantes y } \\
\text { aprender estrategias de afrontamiento } \\
\text { más adaptativas } \\
\text { Planificar la búsqueda de trabajo. } \\
\text { Aprender habilidades asertivas } \\
\text { Mejorar el patrón de sueño }\end{array}$ & $\begin{array}{l}\text { Activación conductual } \\
\text { Pautas de higiene de } \\
\text { sueño } \\
\text { Entrenamiento en } \\
\text { asertividad }\end{array}$ & $\begin{array}{l}\text { AR de bienestar } \\
\text { Actividades gratificantes } \\
\text { AR de interacciones } \\
\text { Lectura de derechos y } \\
\text { responsabilidades asertivas } \\
\text { Lectura de higiene de sueño }\end{array}$ \\
\hline 10 & $\begin{array}{l}\text { Reducir rumiaciones, reducir la } \\
\text { desesperanza y la incertidumbre sobre } \\
\text { su futuro } \\
\text { Aumentar actividades gratificantes y } \\
\text { aprender estrategias de afrontamiento } \\
\text { más adaptativas } \\
\text { Planificar la búsqueda de trabajo. }\end{array}$ & $\begin{array}{l}\text { Activación conductual } \\
\text { Entrenamiento en } \\
\text { respiración } \\
\text { diafragmática }\end{array}$ & $\begin{array}{l}\text { AR de bienestar } \\
\text { Actividades gratificantes } \\
\text { AR de interacciones } \\
\text { AR de respiración (sentado) }\end{array}$ \\
\hline 11 & $\begin{array}{l}\text { Reducir rumiaciones, reducir la } \\
\text { desesperanza y la incertidumbre sobre } \\
\text { su futuro } \\
\text { Aumentar actividades gratificantes y } \\
\text { aprender estrategias de afrontamiento } \\
\text { más adaptativas } \\
\text { Planificarla búsqueda de trabajo. }\end{array}$ & $\begin{array}{l}\text { Activación conductual } \\
\text { Reestructuración } \\
\text { cognitiva }\end{array}$ & $\begin{array}{l}\text { Listado de actividades que le } \\
\text { provoquen bienestar } \\
\text { Actividades gratificantes } \\
\text { AR de respiración (sentado) } \\
\text { AR de sustitución de ideas } \\
\text { irracionales por otras más } \\
\text { realistas }\end{array}$ \\
\hline 12 & $\begin{array}{l}\text { Reducir rumiaciones, reducir la } \\
\text { desesperanza y la incertidumbre sobre } \\
\text { su futuro } \\
\text { Aumentar actividades gratificantes y } \\
\text { aprender estrategias de afrontamiento } \\
\text { más adaptativas }\end{array}$ & $\begin{array}{l}\text { Activación conductual } \\
\text { Reestructuración } \\
\text { cognitiva }\end{array}$ & $\begin{array}{l}\text { Listado de actividades que le } \\
\text { provoquen bienestar } \\
\text { Actividades gratificantes } \\
\text { AR de respiración (sentado) } \\
\text { AR de sustitución de ideas } \\
\text { irracionales }\end{array}$ \\
\hline 13 & $\begin{array}{l}\text { Reducir rumiaciones, reducir la } \\
\text { desesperanza y la incertidumbre sobre } \\
\text { su futuro } \\
\text { Aumentar actividades gratificantes y } \\
\text { aprender estrategias de afrontamiento } \\
\text { más adaptativas } \\
\text { Planificarla búsqueda de trabajo } \\
\text { Mejorar el patrón de sueño }\end{array}$ & $\begin{array}{l}\text { Activación conductual } \\
\text { Pautas de higiene de } \\
\text { sueño }\end{array}$ & $\begin{array}{l}\text { AR de bienestar } \\
\text { Actividades gratificantes } \\
\text { AR de respiración (de pie) } \\
\text { AR de sustitución de ideas } \\
\text { irracionales }\end{array}$ \\
\hline
\end{tabular}




\begin{tabular}{|c|c|c|c|}
\hline 14 & $\begin{array}{l}\text { Reducir rumiaciones, reducir la } \\
\text { desesperanza y la incertidumbre sobre } \\
\text { su futuro } \\
\text { Aumentar actividades gratificantes y } \\
\text { aprender estrategias de afrontamiento } \\
\text { más adaptativas } \\
\text { Aprender habilidades asertivas }\end{array}$ & $\begin{array}{l}\text { Activación conductual } \\
\text { Entrenamiento en } \\
\text { habilidades asertivas }\end{array}$ & $\begin{array}{l}\text { AR de bienestar } \\
\text { Actividades gratificantes } \\
\text { AR de respiración (andando) } \\
\text { AR de interacciones (expresión } \\
\text { de emociones negativas) } \\
\text { AR de sustitución de ideas } \\
\text { irracionales }\end{array}$ \\
\hline 15 & $\begin{array}{l}\text { Reducir rumiaciones, reducir la } \\
\text { desesperanza y la incertidumbre sobre } \\
\text { su futuro } \\
\text { Aumentar actividades gratificantes y } \\
\text { aprender estrategias de afrontamiento } \\
\text { más adaptativas }\end{array}$ & $\begin{array}{l}\text { Activación conductual } \\
\text { Psicoeducación } \\
\text { sexualidad }\end{array}$ & $\begin{array}{l}\text { AR de bienestar } \\
\text { Actividades gratificantes } \\
\text { AR de respiración } \\
\text { (haciendo otra tarea) } \\
\text { AR de interacciones } \\
\text { AR de sustitución de ideas } \\
\text { irracionales }\end{array}$ \\
\hline 16 & $\begin{array}{l}\text { Reducir rumiaciones, reducir la } \\
\text { desesperanza y la incertidumbre sobre } \\
\text { su futuro } \\
\text { Aumentar actividades gratificantes y } \\
\text { aprender estrategias de afrontamiento } \\
\text { más adaptativas } \\
\text { Aprender habilidades asertivas }\end{array}$ & $\begin{array}{l}\text { Activación conductual } \\
\text { Entrenamiento en } \\
\text { habilidades asertivas }\end{array}$ & $\begin{array}{l}\text { AR de bienestar } \\
\text { Actividades gratificantes } \\
\text { AR de respiración (con } \\
\text { activación) } \\
\text { AR de interacciones (expresión } \\
\text { de emociones negativas y hacer } \\
\text { críticas) } \\
\text { AR de sustitución de ideas } \\
\text { irracionales } \\
\text { Lectura de hacer críticas }\end{array}$ \\
\hline 17 & $\begin{array}{l}\text { Reducir rumiaciones, reducir la } \\
\text { desesperanza y la incertidumbre sobre } \\
\text { su futuro } \\
\text { Aumentar actividades gratificantes y } \\
\text { aprender estrategias de afrontamiento } \\
\text { más adaptativas } \\
\text { Aprender habilidades asertivas }\end{array}$ & $\begin{array}{l}\text { Activación conductual } \\
\text { Entrenamiento en } \\
\text { habilidades asertivas }\end{array}$ & $\begin{array}{l}\text { AR de bienestar } \\
\text { Actividades gratificantes } \\
\text { AR de respiración } \\
\text { AR de interacciones } \\
\text { AR de sustitución de ideas } \\
\text { irracionales } \\
\text { Lectura de hacer peticiones }\end{array}$ \\
\hline 18 & $\begin{array}{l}\text { Evaluar el logro de los objetivos } \\
\text { terapéuticos }\end{array}$ & & $\begin{array}{l}\text { AR de bienestar } \\
\text { Actividades gratificantes } \\
\text { AR de respiración } \\
\text { AR de interacciones } \\
\text { AR de sustitución de ideas } \\
\text { irracionales }\end{array}$ \\
\hline $\begin{array}{l}19 \\
\text { Segui- } \\
\text { miento }\end{array}$ & $\begin{array}{l}\text { Evaluar el mantenimiento de las } \\
\text { mejoras }\end{array}$ & $\begin{array}{l}\text { Entrevista abierta } \\
\text { Psicoeducación } \\
\text { sexualidad }\end{array}$ & BDI-II \\
\hline
\end{tabular}

AR: Auto-registro

A fin de obtener medidas para contrastar el posible cambio debido a la intervención, en el seguimiento se repitió la administración del BDI- II aplicado en la segunda sesión de la evaluación de la línea base.

En la tercera sesión el cliente obtuvo una puntuación de 28 (depresión moderada) y en la decimonovena sesión una puntuación de 3 (ausencia de 
depresión).

Por otro lado, puesto que el cliente no completó los auto-registros en todas las ocasiones en las que se prescribieron, fue necesario recurrir a otras estrategias para evaluar la efectividad de la terapia. En concreto, la terapeuta en todas las sesiones fue analizando junto con el cliente las actividades que había realizado en la anterior semana, valorando su dominio y satisfacción. El número de actividades gratificantes que realizaba entre sesión y sesión se muestra de forma gráfica en la Figura 2.

Para poder obtener otras medidas de los cambios debidos a la intervención se ha recogido el número de verbalizaciones negativas (p.ej.: "no me apetece hacer nada", "me quiero quedar en la cama", "no quiero trabajar") y verbalizaciones positivas (p.ej.: "me siento bien", "me siento mejor, más activado, con más ganas de seguir, de hacer cosas") reflejadas por la terapeuta en sus historias clínicas. La Figura 3 recoge ambos tipos de verbalizaciones para poder evaluar la evolución de M. a lo largo de las sesiones.

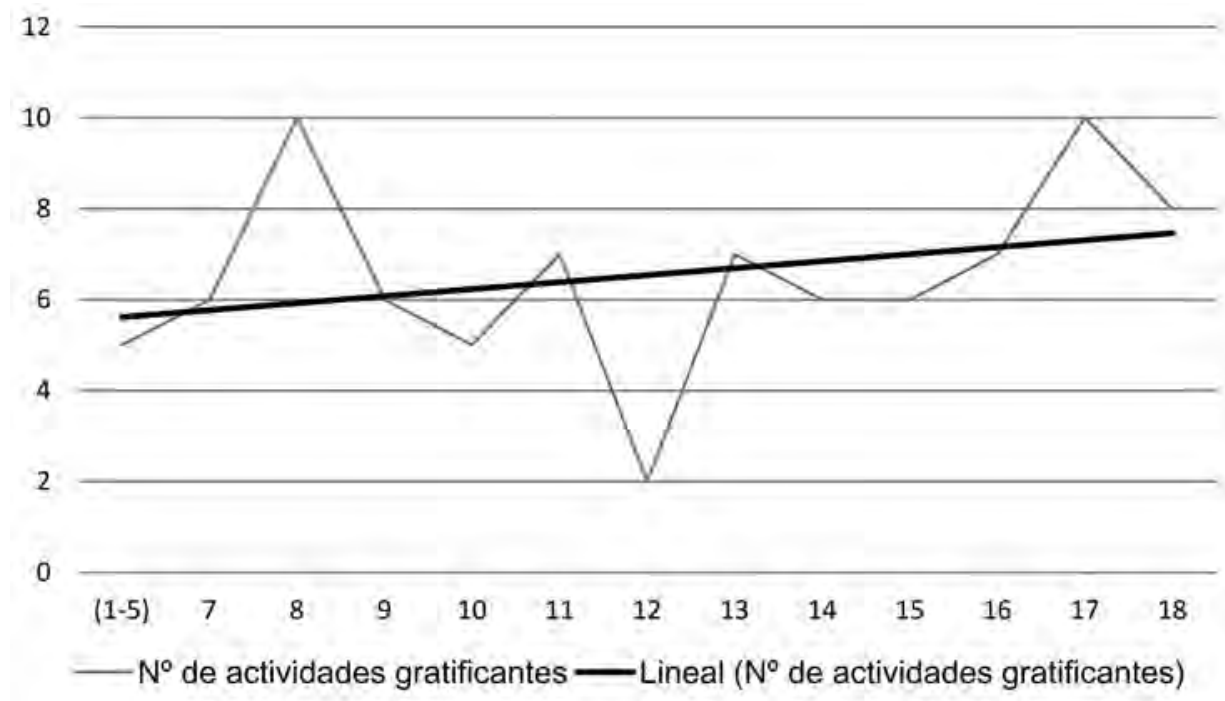

Figura 2. Actividades gratificantes

Los resultados de la intervención pueden apreciarse en la diferencia entre las puntuaciones del autoinforme previas y posteriores a la fase de intervención, en el número de actividades gratificantes, las verbalizaciones negativas y las verbalizaciones positivas.

En la Figura 2 se puede observar cómo el número de actividades gratificantes aumentó y sólo se produjo un descenso en la decimosegunda sesión, cuando el cliente manifestó haber pasado una "mala semana". Este patrón se observa mucho más claro en las verbalizaciones del cliente en las sesiones (Figura 3). Las verbalizaciones positivas aumentaron a lo largo de las sesiones y las verbalizaciones negativas disminuyeron en frecuencia, dándose los mayores picos en las últimas 


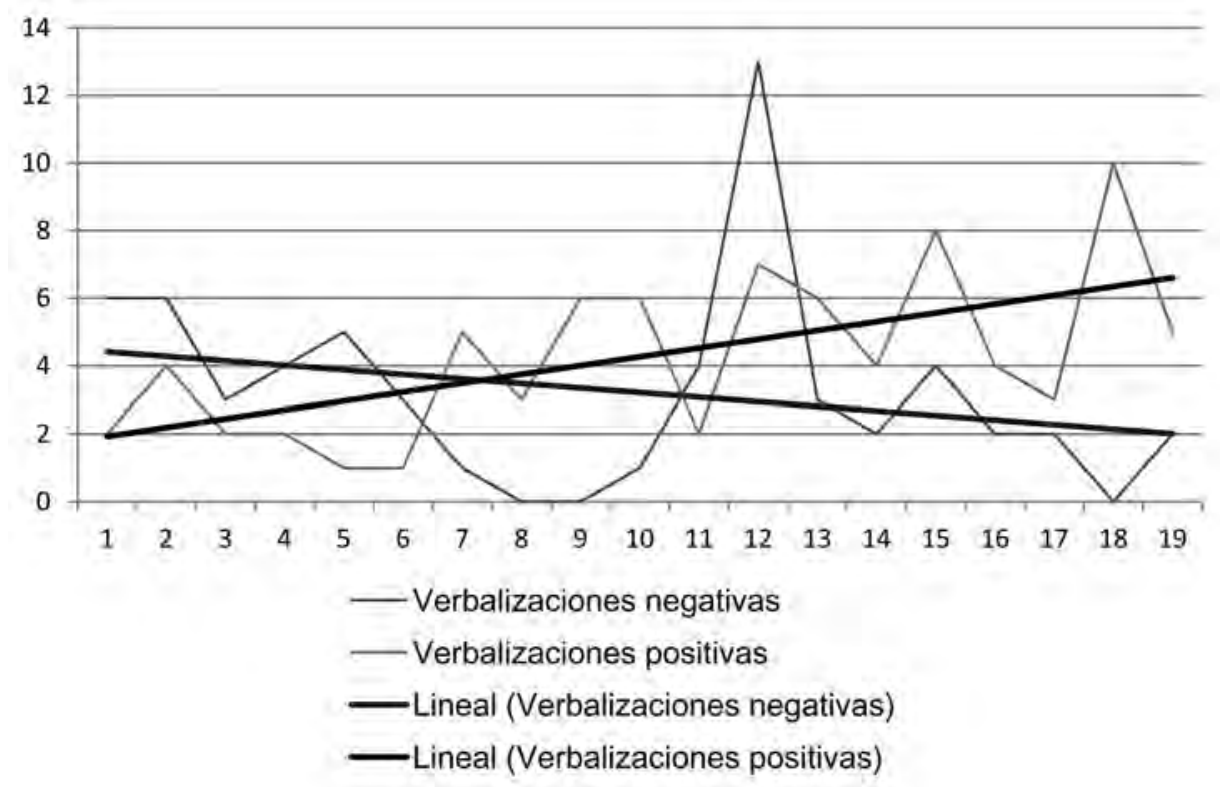

Figura 3. Verbalizaciones negativas y positivas

sesiones (a excepción de la decimosegunda sesión antes mencionada donde este patrón se invierte).

Estos resultados se corresponden con lo planteado, ya que la desaparición progresiva de las conductas de evitación y el refuerzo de las conductas gratificantes y orientadas hacia los valores conduce, a la larga, a una mejora del estado anímico.

En cuanto a la sintomatología ansiosa, el cliente manifestó en las últimas sesiones, y pudo observarse en sus auto-registros, que su sueño había mejorado y que se había reducido el tiempo que dedicaba a las rumiaciones sobre su pasado o sobre su futuro.

Junto con estas medidas, puede señalarse que el cliente expresó su satisfacción por la terapia e informó encontrarse plenamente recuperado. Manifestó que veía claro que había llegado el momento del alta.

\section{Discusión}

El estudio de este caso único nos ha permitido conocer el abordaje terapéutico de un caso con sintomatología ansioso-depresiva. La presencia de distintos síntomas ha otorgado un papel relevante al análisis funcional para la determinación tanto de objetivos como de las técnicas a utilizar para poder alcanzarlos.

De entre las técnicas utilizadas destaca la activación conductual, ya que como nos indicaba el análisis funcional, la conducta problema del cliente se caracterizaba por un patrón evitativo de comportamiento. Se ha podido observar que, en la medida en que M. empezó a tomar contacto con actividades gratificantes orientadas a sus 
valores (conseguir un empleo propio y dedicarse a la música), su estado de ánimo fue mejorando.

El entrenamiento en habilidades de afrontamiento (manejo de la ira, habilidades sociales y la respiración controlada) parece que facilitó el éxito en los demás objetivos, al proporcionar control al cliente en las situaciones difíciles que se le presentaban a lo largo del tratamiento.

Otro cambio que se observó en sesión se produjo en las verbalizaciones del cliente. Estas verbalizaciones fueron registradas por la terapeuta en sus historias clínicas y después cuantificadas para este estudio. A pesar de los posibles sesgos que puede implicar al haber sido recogidas por la propia terapeuta y después categorizadas por un único juez, se decidió incluir esta medida al considerar la conducta verbal como descriptiva de la conducta manifiesta general y, por tanto, indicadora de cambio terapéutico. Después de categorizar y cuantificar las verbalizaciones se puede observar cómo a lo largo de las sesiones se produjo, como ya se ha comentado, un aumento de las verbalizaciones positivas y una disminución de las negativas.

La presente descripción del caso clínico posee una serie de limitaciones, que se deben destacar. Habría resultado oportuno la utilización de cuestionarios para evaluar la sintomatología ansiosa (como por ejemplo el STAI, State Trait Anxiety Inventory, STAI Spielberger, 1970; Seisdedos, 1982) y de auto-registros del cumplimiento de las actividades y de la valoración de su dominio y satisfacción; tales instrumentos hubieran ofrecido una apreciación más exhaustiva de los resultados obtenidos. Tampoco se han podido evaluar los resultados a más largo plazo por la propia gestión del centro, al coincidir el alta con las vacaciones de verano.

$\mathrm{Al}$ emplear un diseño $\mathrm{AB}$ en un entorno poco controlado debemos ser cautos al atribuir un efecto exclusivo de la intervención como fuente de la mejoría del cliente. Se debe ser consciente de que determinados cambios podrían explicarse, en ocasiones, por otras variables del entorno de la persona.

En este sentido, la investigación futura de casos clínicos debe tratar de incorporar otros diseños más complejos que permitan una mayor seguridad en la interpretación de los cambios producidos por la terapia.

\section{Referencias bibliográficas}

American Psychiatric Association. (2000). Diagnostic and statistical manual of mental disorders DSM-IV-TR ( $4^{\mathrm{a}}$ ed. texto rev.). Washington, DC: Author.

American Psychiatric Association. (2013). Diagnostic and statistical manual of mental disorders (5th ed.). Washington, DC: Author.

Aragonés E, Piñol JL, Labad A, Masdéu RM, Pino M, Cervera J. (2004). Prevalence and determinants of depressive disorders in primary care in Spain. Int J Psychiatry Med. 34:21-35.

Beck, A. T. (1967). Depression: clinical, experimental, and theoretical aspects. Nueva York: Harper and Row. Beck, A. T. (1987). Cognitive models of depression. Journal of Cognitve Psychotherapy, 1, 5-37. 
Beck, A. T., Steer, R. A. y Brown, G. K. (1996). Manual for the BDI-II. San Antonio, TX: Psychological Corporation.

Bernstein, D.A. y Borkovec, T.D. (1983): Entrenamiento en relajación progresiva. Bilbao: DDB

Borrell-Carrió F, Caterina-Bargalló M. (2003) Trastornos de salud mental. En: Martín- Zurro A, Cano-Pérez JF, editors. Atención Primaria. Conceptos, organización y práctica clínica, vol. 2. (pp. 1015-46). Madrid: Elsevier

Butler A.C., Chapman J.E., Forman y Beck A.T. (2006). The empirical status of cognitive-behavioral therapy: a review of meta-analyses. Clin Psychol Rev. 26, 17-31.

Carrobles, J. A.. (2011). Trastornos depresivos. En Caballo, V. E., Salazar, I. C., y Carrobles , J. A. Manual de psicopatología y trastornos psicológicos (pp. 311-337). Madrid: Pirámide.

Cuijpers, P., Berking, M., Andersson, G., Quigley, L.,M., Kleiboer, A., y Dobson, K. S., (2013). A meta-analysis of cognitive-behavioural therapy for adult depression, alone and in comparison with other treatments. Canadian Journal of Psychiatry, 58(7), 376-85.

Díaz A, López A, Ruiz-Clavijo M.T., Vargas M.L. (2001). Evolución del consumo de antidepresivos en el área de Zamora desde 1996 a 1999. Aten Primaria, 28, 333-339.

Echeburúa E, Salaberria K, de Corral P, Cenea R, Berasategui T. (2000).Tratamiento del trastorno mixto de ansiedad y depresión: resultados de una investigación experimental. Análisis y Modificación de Conducta.26, 509-535.

Echeburúa E, Salaberria K, de Corral P, Cenea R, Berasategui T. (2006). Treatment of mixed anxiety-depression disorder: long-term outcome. Behav Cogn Psychother. 34, 95-101.

ESEMeD/MHEDEA 2000 Investigators (2004). Disability and quality of life impact of mental disorders in Europe: results from the European Study of the Epidemiology of Mental Disorders (ESEMeD) Project. Acta Psychiatrica Scandinavica, 109, 38-46.

ESEMeD/MHEDEA 2000 Investigators (2004). Prevalence of mental disorders in Europe: results from the European Study of the Epidemiology of MentalDisorders(ESEMeD) Project. ActaPsychiatrica Scandinavica, 109, 21-27

Ferster, C. B. (1965). Classification of behavior pathology. En L. Krasner y L. P. Ullman (dirs.), Research in behavior modification (pp.6-26). Nueva York: Holt, Rinehart and Winston.

Ferster, C. B. (1973). A functional analysis of depression. American Psychologist, 857-870.

Grandes G, Montoya I, Arietaleanizbeaskoa MS, Arce V, Sánchez A. (2011). The burden of mental disorders in primary care. Eur Psychiatry. 26, 428-435.

Haro, J. M., Palacín, C., Vilagut, G. Martínez, M., Bernal, M. Luque, I., Codony, M., Dolz, M., Alonso, J. y Grupo ESEMeD España (2006). Prevalencia de los trastornos mentales y factores asociados: resultados del estudio ESEMED-España. Medicina Clínica, 126, 445-451.

Hollon S.D., Stewart M.O., Strunk D.R. (2006) Enduring effects for cognitive behavior therapy in the treatment of depression and anxiety. Annu Rev Psychol. 57, 285-315.

Hollon, S. D. y Ponniah, K. (2010). A review of empirically supported psychological

Hornillos C. (2009). Presencia física de profesionales de SM en un Centro de AP como forma alternativa de coordinación. Una experiencia piloto. Rev Asoc Esp Neuropsiq. 29(104), 355-370.

Hunsley J., Lee C.M. (2007). Research-informed benchmarks for psychological treatments: eficacy studies, effectiveness studies, and beyond. Prof Psychol: Res Pract. 38, 21-33.

Jauregui, A., Ponte, J., Salgueiro, M., Unanue, S., Donaire, C., et al. (2015). Efficacy of a cognitive and behavioural psychotherapy applied by primary care psychologists in patients with mixed anxiety-depressive disorder: a research protocol. BMC family practice, 16(1), 39.

Labrador, F.J. (1992). El estrés. Nuevas técnicas para su control. Madrid: Ediciones Temas Hoy.

Lewinshon P. M. (1974). A behavioral approach to depression. En R. J. Friedman y M. M. Karz (dirs.). The psychology of depression: contemporary theory and research (pp.157-158). Nueva York: Wiley.

Lewinshon, P. M., Hoberman, H., Teri, L. y Hautizinger, M. (1985). An integrative theory of depression. En S. Reiss y Bootzin, R. R. (Eds.), Theoretical issues in behavior therapy (pp. 331-359). Orlando: Academic Press.

Lewinsohn, P. M. y Gotlib, I. H. (1995). Behavioral theory and treatment of depression. En E.E. Beckham y W.R. Leber (eds.), Handbook depression (pp.329-351). New York: Guilford.

Méndez, F.X., Olivares, J. y Quiles, M.J.(1998). Técnicas de relajación y respiración. En J. Olivares y F.X. Méndez, F.X. (Dir.), Técnicas de modificación de conducta (pp. 27-78). Madrid: Biblioteca Nueva.

Nezu, A. \& Nezu, C. (2008). Entrenamiento en solución de problemas. En V. Caballo (Ed.). Manual de técnicas de terapia y modificación de conducta. Madrid: Siglo XXI. 
Nezu, A. M., Nezu, C. M. y Perri M.G. (1989). Problem-solving therapy for depression: theory, research, and clinical guidelines. New York: Wiley.

Organización Mundial de la Salud. CIE 10 (1992). Décima Revisión de la Clasificación Internacional de Las Enfermedades. Trastornos Mentales y del Comportamiento: Descripciones Clínicas y pautas para el Diagnóstico. Madrid: Meditor.

Pérez Álvarez, M., García Montes, J. M. (2003). Guía de tratamientos psicológicos eficaces para la depresión. En Pérez Álvarez, M., Fernández Rodríguez, C., Amigo Vázquez, I., \& Fernández Hermida, J. R.. Guía de tratamientos psicológicos eficaces. Madrid: Ediciones Pirámide.

Richards D.A., Sycklin R. (2009). Improving access to psychological therapies: Phase IV prospective cohort study. Br J Clin Psychol. 48, 377-96.

Sacco, W. P. y Beck, A.T. (1995). Cognitive theory and therapy. En E.E. Beckham y W.R. Leber (eds.), Handbook depression (pp.329-351). New York: Guilford.

Sanz, J., García-Vera, M. P., Espinosa, R., Fortín, M. y Vázquez C. (2003). Adaptación española del Inventario para la Depresión de Beck-II (BDI-II): 3. Propiedades psicométricas en pacientes con trastornos psicológicos. Clínica y Salud, 16, 121- 142.

Sanz, J., Perdigón, A. L. y Vázquez C. (2003). Adaptación española del Inventario para la Depresión de Beck-II (BDI-II): 2. Propiedades psicométricas en población general. Clínica y Salud, 14, 249-280.

Seligman, M. (1981). Indefensión. Madrid: Debate (orig. 1975).

Serrano-Blanco A, Palao DJ, Luciano JV, Pinto-Meza A, Luján L, Fernández A, et al. (2010). Prevalence of mental disorders in primary care: results from the diagnosis and treatment of mental disorders in primary care study (DASMAP). Soc Psychiat Epidemiol. 45, 201-210.

Skinner, B. F. (1953). Science and human behavior. Nueva York: MacMillan.

Spielberger, C., Gorsuch, R. y Lushene, R. (1970). Manual for the State Trait Anxiety Inventory. Palo Alto, California, Consulting Psychologist Press. (Adapt. Española, TEA, 1982). Versión española, Seisdedos, N. (1982), Madrid: TEA Ediciones.

Spielmans G.I., Berman M.I., Usitalo A.N. (2011) Psychotherapy versus second-generation antidepressants in the treatment of depression: a meta-analysis. J Nerv Ment Dis., 199,142-149.

Vallejo J, Bataller R. (2000). Relación entre los estados de ansiedad y los estados depresivos. En: Vallejo J, Gastó C, editors. Trastornos afectivos: ansiedad y depresión. ( p. 724-761). Barcelona: Mason.

Vallejo Pareja, M. Á. y Comeche Moreno M. I (2012). Depresión. En Vallejo Pareja, M. Á. (Coord.). Manual de terapia de conducta ( $2^{\mathrm{a}}$ edición). Madrid: Dykinson.

Van Orden M., Hoffman T., Haffmans J., Spinhoven P.Y., Hoencamp E. (2009). Collaborative Mental Health Care versus Care as Usual in a Primary Care Setting: a randomised controlled trial. Psychiatr Serv. 60(1), 74-79. doi: 10.1176/appi.ps.60.1.74. 